\title{
QUALITY OF CARE: A COMPARISON OF PREFERENCES BETWEEN MEDICAL SPECIALISTS AND PATIENTS WITH CHRONIC DISEASES
}

\author{
MARIEKE A. E. VAN DER WAAL.' ANTON F. CASPARIE' and \\ CHRISTIAAN J. LAKO
}

Institute of Health Care Policy and Management. Faculty of Medicine and Health Sciences, Erasmus University. Rotterdam, The Netherlands and Department of Public Administration and Management Sciences, Nijmegen University. Nijmegen. The Vetherlands

\begin{abstract}
In this study, we have looked for differences between medical specialists and patients with chronic diseases (COPD, rheumatoid arthritis and diabetes mellitus) in preferences of aspects of care in relation to the quality of care. Firstly, to enumerate relevant aspects for chronic diseases, open interviews and a concept mapping were conducted among patients with a chronic disease. and medical specialists treating ihem. Herc. the respondents have been asked to evaluate statements in relation to the quality of care. Secondly, a final questionnaire, including statements on nine relevant aspects of care, was presented to patients and medical specialists. The response rate among patients was $96 \%(N=260)$ and among medical specialists $67 \%$ $(N=340)$. Both study populations ranked 'effectiveness of care' the highest. However. the difference in opinion between the two populations was significant, mainly due to the patient's giving a higher ranking to 'continuity of care' and a lower ranking to 'efficiency'. Significant differences were also found between the three patient groups on the aspects 'knowledge' and 'waiting time for treatment'. Patients with rheumatoid arthritis ranked 'knowledge' higher and 'waiting time for treatment' lower than did the other two patient groups. A lower level of education, having state-regulated health insurance and being older were associated with a higher preference for 'continuity'. Between the three groups of the medical specialists, no significant differences were found regarding to the profession. age and sex. In conclusion: the patients and medical specialists researched did not show wide differences of opinion on preferences of care in relation to quality. The only exception to this concerned 'continuity of care' which was ranked higher by patients.
\end{abstract}

Key words-quality of care, chronic diseases. preferences for aspects of care. medical specialists. patient with chronic diseases

\section{INTRODUCTION}

Over the last decade, a wide range of activities have been performed in order to assure and, where possible. to improve the quality of health care [1]. The need for cost containment has been accompanied by a tendency to further the quality of health care. In addition, more attention is being paid to the contribution that patients can make to improve the quality of care [1]. This interest also reflects the need for more democratic relations between the various actors involved in health care. In the Netherlands, quality of health care policy is explicitly considered the joint responsibility of providers, patients and insurers [2]. The expectations and opinions of these parties have, therefore, to be geared to each other before quality of care can be effectively assessed and improved.

In this study, we have measured and compared the preferences for aspects of care of both physicians and patients. These preferences represent the attitude of a respondent towards our object of research: aspects of care. The study population has been limited to patients suffering from chronic diseases and the medical specialists treating them. As the number of people suffering from a chronic disease in the Netherlands is steadily increasing [3], it has become imperative to discover what these chronically ill patients think is important in coping with their own future. The chronically ill can, furthermore. be deemed as having considerable experience with the health care system and, as such, they are experts on their own disease. Therefore. these patients are not only able to evaluate the behaviour of health professionals, but they are also able to evaluate some technical aspects of care. We were, moreover, also interested to discover whether potential differences in preferences within the physician and patient study populations were. in some way, related to personal characteristics or to the type of disease.

The results of this study can be of interest for the following reasons. Firstly, through this study potential discrepancies in preferences between physicians and patients with chronic diseases will become apparent. These findings could then facilitate any possible adjustments in health care being made at both the national level, and at the level of institutions and individual providers. Secondly, from a theoretical point of view $[4,5]$, it would be interesting to learn 
whether the difficult illness trajectory management in caring for the chronically ill results in differences between patients and physicians in preferences towards the quality of care.

In the literature only a few studies have been found in which the preferences of patients are compared with those of other concerned parties. Yet none have employed a joint formulation of important aspects. In a study by Smith and Armstrong, patients were asked to compare 20 aspects independently formulated by government and patients [6]. In research by Batalden ef al., patients and medical specialists were asked in separate brainstorming sessions to list the aspects of quality of hospital care they considered important although, in this study, they were not asked to indicate which aspects they felt should been given the highest priority [7]. Recently, Hares et al. [8] have examined the preferences of both small groups of diabetes patients and small groups of providers using a nominal group technique. Here, each sub-group was studied separately.

The study addressed the following questions: Which differences in preferences towards quality of care do exist between patients and physicians, what is the relative importance of aspects of quality of care formulated by both groups and which factors might attribute to these differences?

A problem that could arise in formulating preferences is how to define the various terms and aspects used by physicians and patients. To minimalize this problem we have employed the method of concept mapping between patients and physicians to gain an unambiguous framework of notions [9]. By asking the relative importance of a particular number of statements, the differences in preferences between the respondents can be quantified.

\section{METHODS AND SUBJECTS}

\section{Studv' design}

This study was conducted in four phases. In the first phase, 43 open interviews with medical specialists and patients were held in order to generate relevant aspects of care related to the quality of care of chronic diseases. Interviews were held with four rheumatologists, four diabetes specialists, four pulmonary specialists, nine patients with rheumatoid arthritis, six with COPD and 16 with diabetes mellitus type II.

In the second phase, concept mapping was used for defining the notions on the various aspects, and to determine their relative importance and priority [9]. In the first stage of concept mapping a brainstorming session was held where participants are asked to enumerate various items related to the quality of care. In the second stage, these statements are organized by the respondents into clusters using a computer programme. This programme is based on Principle Components Factor Analysis which generates the clusters of statements and was first developed by Trochim [10]. In the third stage, these clusters were labelled and placed on a priority scale. We asked three medical specialists and three patients to participate in this phase of this study. The concept mapping resulted in twenty different statements and related aspects of health care.

The underlying method of the final questionnaire is the method of pairwise comparison. The use of 20 aspects would, according to the formula $n(n-1) / 2$ developed by Bock and Jones [11], result in 190 pairs of aspects for comparison, an impossible task. A suitable design, developed by Cochran and Cox [12], was found for presenting our final statements to respondents [12]. In this design, only nine statements can be used for comparison. Therefore, in the third phase, a reduction of aspects had to be made and the Partial Balanced Incomplete Block Design (PBIB 13,1 Bock and Jones) has been applied [11]. For reduction a group of 54 undergraduates in health administration were asked to judge which statements matched best with the notion 'quality of care'. Essential is that judges have a certain level of intelligence and abstraction [13] and their task is simply to consider which statements are more appropriate. To reduce the number of statements, the discriminal dispersion of each statement was first computed. This can be compared with statistical variance. A statement with a high discriminal dispersion is one where a wide variation is found between the judgements. Some of the 20 statements were judged equally by our judges and those with the highest discriminal dispersion were discarded. After reduction the questionnaire has been constructed, which comprised not only the nine concrete statements but also more general information.

In the fourth and final phase, respondents - chronically ill patients and their physicians-were interviewed and the resulting data analyzed.

\section{Data collection}

The nine statements were presented to the respondents in 12 blocks (each block contained 3 different statements) in accordance with the Balanced Incomplete Block Design of Cochran and Cox [12]. In this method, each statement is presented three times, constantly in comparison with two other statements. In each of the 12 blocks respondents were asked to give the three statements a priority number of 1,2 or 3 ( 1 being the most important).When this had been completed, respondents could also add aspects of care or statements they had missed in the questionnaire.

\section{Subjects}

At 22 out-patient departments of 11 General hospitals, 271 patients were asked to fill in the questionnaire before seeing their physician. The criteria for selection were as follows: respondents had to be between 18 and 75 years of age and have been suffering from diabetes mellitus (ICD-code 250), COPD (ICD code $490-496$ ) or rheumatoid arthritis (ARA-criteria) for two years or more. Patients were asked their age, sex, level of education, the duration of 
Table 1. Aspects and corresponding statements as were used in the questionnaire

\begin{tabular}{ll}
\hline Aspect & \multicolumn{1}{c}{ Statement } \\
\hline Waiting time for treatment & A patient who can visit his doctor soon after making an appointment \\
Autonomy & A patient who can make choices between different types of treatment \\
Continuity & Secing the same care provider \\
Patient-physician relationship & A care provider with enough time for the patient \\
Efficiency & A doctor who does not use inappropriate additional diagnostic interventions \\
Effectiveness & A treatment with a favourable effect on the disease \\
Knowledge & A doctor with enough knowledge of chronic diseases \\
Information & A patient who gets all the information needed regarding his health status and treatment \\
Empathy & A provider who tries to understand a patient \\
\hline
\end{tabular}

their disease and their type of health insurance cover. The questionnaire was handed out personally by the researcher. Medical specialists were contacted through the register of their respective National Scientific Societies. Every registered rheumatologist, diabetes specialist and pulmonary specialist practising in a Dutch General hospital was mailed an identical questionnaire $(N=505)$. The questionnaire also contained questions of a more general nature such as the age and sex of the respondent.

\section{Data analysis}

The degree of individual intransitivity (failing to answer consistently) was measured as a percentage [14]. The number of circular triads (a method to determine the seriousness of the intransitivity) was measured according to the design developed by the Meerling group [15]. Bock and Jones' $\chi^{2}$ test [11] was used to determine differences between study populations and the $\chi^{2}$ test of Edwards [14] for variations within study populations. The level of significance was determined at $P<0.05$.

\section{RESULTS}

The remaining nine central aspects, with the accompanying statements as used in the questionnaire. are presented in Table 1.

The response rate for the patients was $96 \%$ $(N=260)$ and for the medical specialists $67 \%$
$(N=340)$. The response rate for rheumatologists was $80 \% \quad(N=77)$, for pulmonary specialists $64 \%$ $(N=137)$ and for diabetes specialists $65 \%(N=126)$. These last two groups were sent a reminder. Thirteen questionnaires were incomplete and were, therefore. not included in our analysis. Table 2 shows some general characteristics of the patients. Among the pulmonary specialists $(N=132), 86 \%$ were male and the mean age was 45 years $(S D=7)$. Among the rheumatologists $(N=72), 78 \%$ were male and the mean age was 44 years $(S D=8)$. The mean age of the diabetes specialists $(N=123)$ was 47 years $(\mathrm{SD}=8)$ and $92 \%$ of them were male.

Using nine statements, the maximum amount of intransitive choices a respondent can make is 30 according to Kendall's formula: $\left(n^{3}-n\right) / 24$ when $n$ is odd (see Refs [14] and [16]). This number is equal to the maximum amount of circular triads that can be made $\left(d_{\max }\right)$. For example, if statement $A$ is preferred above statement $B$ and $B$ is preferred above $C$, then a transitive choice is made when $A$ is preferred above $C$. If $C$ was preferred above $A$, an intransitive choice has been made and, in this case, results in one circular triad. No intransitive choices were made by $36 \%$ of specialists and $17 \%$ of the patients. Seventy-eight percent of the patients and $97 \%$ of the specialists made $\leq 5$ intransitive choices. For $22 \%$ of the patients and $3 \%$ of the specialists, the number of circular triads $(d)$ has been computed as suggested by the Meerling group [15]. The formula for computing $d$, and an example,

Table 2. Characteristics of the patient population

\begin{tabular}{lccc}
\hline Patients with & COPD & Rheumatoid arthritis & Diabetes mellitus \\
& $N=74$ & $N=92$ & $N=94$ \\
\hline Mean age in years & $55(\mathrm{SD}=16)$ & $56(\mathrm{SD}=13)$ & $52(\mathrm{SD}=17)$ \\
Men & 57 & 35 & 55 \\
Women & 43 & 65 & 45 \\
Duration of disease & & & 30 \\
$2-5$ years & 35 & 42 & 23 \\
$5-10$ years & 16 & 25 & 47 \\
$>10$ years & 49 & 33 & 50 \\
Level of education & & & 17 \\
Low & 46 & 47 & 33 \\
Medium & 18 & 25 & 71 \\
High & 36 & 28 & 29 \\
Health insurance & & & \\
Public insurance & 54 & 67 & 33 \\
Private insurance & 46 & &
\end{tabular}

All data are percentages except those for age 
Table 3. Ranking order of aspects with scale values of patients and medical specialists (from high to low)

\begin{tabular}{llll}
\hline Patients $N=255$ & \multicolumn{3}{l}{ Medical specialists $N=326$} \\
\hline Effectiveness & $(1.36)$ & Effectiveness & $(1.64)$ \\
Continuity & $(1.09)$ & Knowledge & $(1.47)$ \\
Knowledge & $(1.08)$ & Information & $(0.93)$ \\
Information & $(0.91)$ & Patient-physician relation & $(0.90)$ \\
Patient-physician relation & $(0.73)$ & Empathy & $(0.78)$ \\
Empathy & $(0.58)$ & Continuity & $(0.59)$ \\
Waiting time for treatment & $(0.17)$ & Efficiency & $(0.49)$ \\
Autonomy & $(0.06)$ & Waiting time for treatment & $(0.21)$ \\
Efficiency & $(0.00)$ & Autonomy & $(0.00)$ \\
\hline
\end{tabular}

are given in Appendix $\mathrm{A}$. If $1-d / d_{\max } \leq 0.5$, the answering pattern of respondents is inconsistent [16] and these data are removed from the data-set. Computing the number of circular triads resulted in the exclusion of five patients and one pulmonary specialist from further analysis because of their high rates of inconsistency.

Before the difference in preference between patients and physicians can be examined, the difference in ranking order of the aspects within the patient population, and within the specialist population, must be defined. The purpose of this test is to discover whether the nine aspects differ from each other on the scale or not. If there is no difference at all between the aspects on the scale then further analysis would not be justifiable. Based on $P<0.05$, according to the $\chi^{2}$ test of Edwards, we can assume that the difference between the aspects on the ranking scale within the patient population and the specialist population must be $>0.12$ and 0.10 , respectively. From Table 3 (view each column separately) we can conclude that most aspects vary in this study and these results have no implications for further analysis.

The preferences of statements, expressed here as aspects, between the population of patients and physicians are shown in Table 3 . The difference between both study populations was significant $\left(\chi^{2}=\right.$ 182.7, $P<0.005$ ), and can be mainly attributed to differences in priority given to the aspects "continuity' and 'efficiency'. Patients are much more likely to emphasize "continuity of care" while physicians give a higher priority to 'efficiency' than do their patients. In Table 3 , the scale values are shown only to compare the priority ranking within both the patient and the specialist study population. It is unacceptable to compare the scale values between the two populations [11].

Between the three groups of patients significant differences in preference were found $\left(\chi^{2}=37.7\right.$, $P<0.005$, Table 4$)$. This disparity is partly due to differences in the aspects 'knowledge' and "waiting time for treatment'. Further, younger patients (47 years and under) showed a stronger preference for 'information' than did older patients $\left(\chi^{2}=32.6\right.$, $P<0.05$ ). No differences based upon gender were found among patients, while those having a lower level of education and state-regulated health care insurance (eligibility for this type of insurance is based upon income and $65 \%$ of the Dutch population fall into this category) showed more preference for 'continuity' $\left(\chi^{2}=138.4, \quad P<0.005 \quad\right.$ respectively $\quad \chi^{2}=38.5$, $P<0.05$ ). The duration of the disease would seem to have no discernible influence.

Among the physicians, no significant differences in ranking order of aspects were found in relation to the specialization, age or sex of the respondent.

The questionnaire also offered respondents the opportunity to add on any additional statements to our list. Thirty-four of the 260 patients ( $13 \%$ ) and 114 of the 327 medical specialists $(35 \%)$ did so. Most statements concerned better cooperation between home and institutional care, patient compliance and accessibility to physicians and medical institutions.

\section{DISCUSSION}

One of the most remarkable results of this study is the high degree of agreement in preferences between medical specialists and chronically ill patients. Both groups gave the highest ranking to 'effectiveness', 'a

Table 4. Ranking order of aspects with scale value of patients with rheumatoid arthritis, COPD and diabetes mellitus (from high to low)

\begin{tabular}{lllll}
\hline $\begin{array}{l}\text { Patients with rheumatoid arthritis } \\
N=90\end{array}$ & $\begin{array}{l}\text { Patients with COPD } \\
N=73\end{array}$ & \multicolumn{2}{l}{$\begin{array}{l}\text { Patients with diabetes mellitus } \\
N=92\end{array}$} \\
\hline Effectiveness & $(1.42)$ & Effectiveness & $(1.42)$ & Effectiveness \\
Knowledge & $(1.27)$ & Continuity & $(1.05)$ & Continuity \\
Continuity & $(1.16)$ & Knowledge & $(1.05)$ & Information \\
Information & $(0.82)$ & Information & $(0.88)$ & Knowledge \\
Physician-patient relation & $(0.72)$ & Physician-patient relation & $(0.55)$ & Physician-patient relation \\
Empathy & $(0.55)$ & Empathy & $(0.49)$ & Empathy \\
Autonomy & $(0.13)$ & Waiting time for treatment & $(0.20)$ & Waiting time for treatment \\
Efficiency & $(0.02)$ & Eficiency & $(0.10)$ & Autonomy \\
Waiting time for treatment & $(0.00)$ & Autonomy & $(0.00)$ & Efficiency \\
\hline
\end{tabular}


treatment that has a favourable effect on the disease'. Only the aspects 'continuity' (seeing the same care provider) and 'efficiency' were rated very differently by the two populations. 'Continuity' in this sense is obviously of more interest for patients than for providers. In studies conducted in service industries, continuity is not mentioned as a determinant of the quality of service and this aspect seems then rather specific to the health care sector [2]. In the study of Smith and Armstrong, 'continuity' was also ranked highly by patients [6] but, in contrast to the findings of Hares et al. [8], the physicians in our study ranked 'continuity' fairly low. The importance of 'continuity' might be explained by the illness trajectory model developed by Strauss. This theory encompasses not only the physiological unfolding of a patient's disease but the total organization of 'work' done during its course [5]. Illness trajectory management has been shown to be difficult in the case of chronic illness because of its long term unpredictable course, and because no single health care provider has responsibility to manage the illness trajectory (that is the multiple clinical interventions provided in varying combinations and in varying settings over the course of illness as the patient fluctuates between acute episodes and more stable periods) [4]. As a consequence chronically ill patients and their families are left with the 'work' of treatment. Therefore, their interest in the clinical decision-making process is great. More 'continuity of care' furthers the participation of patients in the clinical decision-making process.

One unexpected finding was the low ranking given by patients for 'autonomy' (having the opportunity to choose between different types of treatment). It may be that these choices are not important for chronic patients which would make this statement unrepresentative for the notion 'autonomy". The patients in Hares' et al. study ranked autonomy fairly high but the statements mentioned in this study were more concerned with the prerequisites for being able to act autonomously [8]. In contrast to the findings of Smith and Armstrong [6], 'knowledge' of the physician was given a high priority by both the doctors and patients in our study.

The preferences within each of the two study populations were rather consistent. The order of ranking by patients was only partly influenced by the type of disease and indicators of socio-economic status such as level of education and type of health insurance. We might deduce that the lower the socio-economic status, the more important 'continuity of care' becomes. We also found that patients with rheumatoid arthritis rank 'knowledge' (of the physician) higher and 'waiting time for treatment' lower than the two other groups of patients. This could perhaps be explained by the high degree of knowledge and insight available on COPD and diabetes treatment compared to the present scant knowledge on the causes and effective treatments of rheumatic diseases.
Among the specialists, neither their particular specialization nor their age and sex had any discernible influence.

It might be argued that doctors and patients may have differing interpretations of the same statement. Firstly, we would point out that these statements were formulated and agreed upon by both the doctors and patients meeting in joint session. Secondly, the number of intransitive choices present in our study would be considered relatively low according to the criteria laid down by Bezembinder [16]. Therefore, it is concluded that there are no major differences in interpretation between patients and physicians.

As suggested by Wensing et al. [1], both physicians and patients were involved in selecting the aspects of care to be examined. This procedure produces more valid results. A disadvantage of our study might be that the variety of opinions has been limited by the number of participants in the concept mapping procedure as this is lower than what is normally suggested [9]. For the reduction of the aspects students have been selected to judge which statements match best with the notion 'quality of care'. The advantage is that they have the necessary level of intelligence and abstraction to consider which statements are more appropriate [13]. The disadvantage is that these judges are mostly not patients. In the final questionnaire, we used concrete statements and not abstract notions which, we feel, make the answers more valid. A further indication of the content validity comes from the additional statements mentioned by respondents at the end of the questionnaire. Only an average of $25 \%$ of respondents gave any additional items. The most of ten mentioned were cooperation between providers. patient compliance and accessibility to physicians and medical institutions.

The method of pairwise comparison has both advantages and disadvantages. On the positive side. it forces the respondents to make an explicit choice between different aspects as well as offering the researcher the possibility to quantify the results. In addition. the final position of each aspect on the scale is a result of the independent comparison of each statement with the other statements. Another advantage is that, when used in combination with this study design, it simplifies the identification of competitive aspects on the major ranking scale. It gives more information about the reliability of the scale. As mentioned earlier, we were able to determine the discriminal dispersion of each of the original 20 aspects of care. As is common with pairwise comparison, the position of each aspect on the formulated scale was also computed. Two aspects are competitives when they have almost the same scale value. In other words, their position on this scale is almost the same. Therefore, the discriminal dispersion is necessary to be able to delete one of the competitives where opinions on it are unequally divided through the study population. One disadvantage of this method is that it is only effective when used on a limited number of 
statements. The consequence of this reduction is that the instrument might lack relevant statements which then might effect the validity of the instrument.

More research is needed to discover what patients exactly prefer within the statements 'seeing the same care provider' and 'a doctor with enough knowledge of chronic diseases. This can be achieved by presenting various statements about one aspect to respondents.

In conclusion: patients with chronic diseases and medical specialists do not vary considerably in their opinions on preferences of care in relation to quality of care. However, medical specialists must realize that, for the chronically ill, 'continuity of care' (always seeing the same doctor) is one of the most important aspects of the quality of care.

Acknowledgements-The authors wish to express their gratitude to Peter Harteloh MD for conducting the open interviews, and Professor Willem Heiser of Leiden University, the Netherlands, for his advice and support during data analysis. This study was funded by a grant from the Dutch Ministry of Welfare, Health Care and Culture.

\section{REFERENCES}

1. Wensing M., Grol R. and Smits A. Quality judgements by patients on general practice care: a literature analysis. Soc. Sci. Med. 38, 45, 1994.

2. Casparie A. F. View from the Netherlands. Qual. Hlth Care 2, 138, 1993.

3. van Lenthe F. J. Prevalentie-analyse tan een aantal chronische ziekten tussen 1990-2005. Rijksinstituut voor Volksgezondheid en Milieuhygiëne. Bilthoven. 1991.

4. Lehr $\mathbf{H}$. and Strosberg M. Quality improvement in health care: is the patient still left out? $Q R B 17,326$, 1991.

5. Strauss A., Fagerhaugh S., Suczek B. and Wiener C. Social Organization of Medical Work. The University of Chicago Press, Chicago, 1985.

6. Smith C. H. and Armstrong D. Comparison of criteria derived by government and patients for evaluating general practitioner's services. $B r . M e d . J .299,494$. 1989

7. Batalden P. B. and Nelson E. C. Hospital quality: patient, physician and employee judgements. $I n t . J$. Hlth Qual. Assoc. 3, 7, 1991

8. Hares T. et al. Diabetes care: who are the experts? Qual. Hlth Care 1, 219, 1992.

9. Trochim W. M. K. An introduction to concept mapping for planning and evaluation. Eval. Program Plann. 12, 1. 1989.

10. Trochim W. K. M. The Concept System. Computer Program. Ithaca, New York 1987/1989.

11. Bock R. D. and Jones L. V. The Measurement and Prediction of Judgement and Choice. Holden-Day, San Francisco, 1967

12. Cochran W. C. and Cox G. M. Experimental Designs, 2nd Edn. John Wiley and Sons, New York, 1957.

13. Swanborn P. G. Schaaltechnieken: theorie en praktijk tan acht eenvoudige procedures. Boom, Meppel, 1982.

14. Edwards A. L. Techniques of Attitude Scale Construction. Appleton-Century-Crofts, New York. 1957.
15. The Meerling Group. Methoden en technieken tan psychologisch onderzoek. Deel 1: Model, observatie en beslissing. Boom, Meppel, 1981.

16. Bezembinder T. G. G. Van rangorde naar continuum. Een verhandeling over datastructuren in de psychologie. Van Lochum Slaterus, Deventer, 1970.

\section{APPENDIX A}

Two examples for computing the amount of circular triads (d) and the amount of inconsistency according to Edwards

Example 1: Comparative judgements for a judge with no circular triads

\begin{tabular}{lcccccc}
\hline Statement & 1 & 2 & 3 & 4 & 5 & 6 \\
\hline 1 & - & 1 & 1 & 1 & 1 & 1 \\
2 & 0 & - & 1 & 1 & 1 & 1 \\
3 & 0 & 0 & - & 1 & 1 & 1 \\
4 & 0 & 0 & 0 & - & 1 & 1 \\
5 & 0 & 0 & 0 & 0 & - & 1 \\
6 & 0 & 0 & 0 & 0 & 0 & - \\
\hline $\mathrm{a}$ & 0 & 1 & 2 & 3 & 4 & 5 \\
$\mathrm{a}^{2}$ & 0 & 1 & 4 & 9 & 16 & 25 \\
\hline
\end{tabular}

$a=$ sum of the entries in a given column.

Example 2: Comparative judgements for a judge with circular triads

\begin{tabular}{lcccccc}
\hline Statement & 1 & 2 & 3 & 4 & 5 & 6 \\
\hline 1 & - & 1 & 1 & 0 & 1 & 0 \\
2 & 0 & - & 1 & 1 & 0 & 1 \\
3 & 0 & 0 & - & 0 & 1 & 0 \\
4 & 1 & 0 & 1 & - & 1 & 1 \\
5 & 0 & 1 & 0 & 0 & - & 1 \\
6 & 1 & 0 & 1 & 0 & 0 & - \\
\hline $\mathrm{a}$ & 2 & 2 & 4 & 1 & 3 & 3 \\
$\mathrm{a}^{2}$ & 4 & 4 & 16 & 1 & 9 & 9 \\
\hline
\end{tabular}

$a=$ sum of the entries in a given column.

The number of circular triads will be given by:

$$
d=\left\{\left(\frac{1}{12}\right)(n)(n-1)(2 n-1)\right\}-\left\{\frac{1}{2} \Sigma a^{2}\right\}
$$

The maximum number of circular triads will be given by:

$$
d_{\max }=\frac{\left(n^{3}-n\right)}{24}
$$

when $n$ is odd or

$$
d_{\max }=\frac{\left(n^{3}-4 n\right)}{24}
$$

when $n$ is even. In both examples

$$
d_{\max }=\frac{\left(6^{3}-4 * 6\right)}{24}=8
$$


In the first example:

$$
\begin{aligned}
d & =\left\{\left(\frac{1}{12}\right) *(6) *(6-1) *(2 * 6-1)\right\} \\
& -\left\{\frac{1}{2} *(0+1+4+9+16+25)\right\}=27.5-27.5=0
\end{aligned}
$$

and so

$$
1-d / d_{\max }=1
$$

and the conclusion is that the answering pattern of this respondent is consistent.
In the second example:

$$
\begin{aligned}
d= & \left\{\left(\frac{1}{12}\right) *(6) *(6-1) *(2 * 6-1)\right\} \\
& -\left\{\frac{1}{2} *(4+4+16+1+9+9)\right\}=27.5-21.5=6
\end{aligned}
$$

and in this case

$$
1-d / d_{\max }=0.25
$$

and the conclusion is that the answering pattern of this respondent is inconsistent. 